\title{
Racismo y Bienestar: la hibridación del movimiento eugenésico
}

\author{
Racism and Welfare: The Hybridization of Eugenics Movement
}

\author{
Jesús Parra Sáez \\ Universidad de Murcia \\ jesus.parra@um.es \\ ORCID: 0000-0003-2301-2963
}

Recibido: 4-5-2018

Aceptado: 10-6-2018

Cómo citar este artículo / Citation: PARRA SÁEZ, Jesús (2018). Racismo y Bienestar: la hibridación del movimiento eugenésico. Pasado y Memoria. Revista de Historia Contemporánea, 17, pp. 211-233. https://doi.org/10.14198/PASADO2018.17.08

\section{Resumen}

El milenario interés del ser humano por mejorar sus atributos naturales derivó a finales del siglo XIX en la emergencia de la 'eugenesia' como ciencia que estudiaba la mejora del linaje humano. En la práctica, el movimiento ideológico y político eugenésico se materializó en la primera mitad del siglo XX, especialmente en Reino Unido, Estados Unidos y Alemania. Estos tres Estados, considerados como la línea principal del movimiento eugenésico, intentaron llevar a cabo la pretendida mejora de la especie humana a través de toda una serie de políticas de carácter homófobo y racista cuya consecuencia directa fue la esterilización involuntaria e incluso el asesinato de miles de personas. Sin embargo, el final de la II Guerra Mundial supuso un punto de inflexión en el movimiento eugenésico, el cual fue modificando progresivamente su carácter racista para desarrollar su idea de perfeccionamiento humano desde el punto de vista del "bienestar de la sociedad" y la mejora de la calidad de vida de los ciudadanos, dando lugar a una hibridación racismo-bienestar en la ideología eugenésica de segunda mitad del siglo XX.

Palabras clave: Eugenesia. Racismo. Hibridación. Estado del Bienestar. Homicultura. Puericultura. Movimiento eugenésico. 


\begin{abstract}
Human beings millenarian interest in improving their natural attributes culminated at the end of the 19th century with the emergence of 'eugenics' as a science that studied the enhancement of human lineage. In practice, the ideological and political eugenics movement materialized in the first half of the 20th century, especially in the United Kingdom, the United States of America and Germany. These three nations, embodying the eugenics movement mainstream, tried to carry out the desired improvement of human species by applying several homophobic and racist policies whose direct consequence was involuntary sterilization and murder of thousands of people. However, the end of the Second World War brought about a turning point for the eugenics movement. It gradually modified its racist nature in order to develop the idea of human enhancement from the point of view of "social welfare" and the improvement of citizens' quality of life, giving rise to a racism-welfare hybridization within the eugenics ideology of the second half of the 20th century.
\end{abstract}

Keywords: Eugenics. Racism. Hybridization. Welfare State. Homiculture. Puericulture. Eugenics Movement.

\title{
1. Introducción
}

La preocupación del ser humano por la mejora de su propia especie tiene un origen incierto. Los estudiosos de su desarrollo no han dudado en señalar al siglo V a.C. -concretamente al sistema educacional espartano de la agogé- como el inicio de las estrategias humanas con tal fin, aunque los primeros vestigios de este interés pueden rastrearse más de dos mil años atrás en el tiempo en el seno de la civilización sumeria. Sin embargo, fue a partir de 1883 de mano de Francis Galton y gracias al firme apoyo de la ciencia de la época, cuando dichas estrategias alcanzaron una importancia vital en los ámbitos médico y político. En la investigación acerca de la mejora humana y el establecimiento de la eugenesia en el siglo XIX como ciencia que estudia tales menesteres, diversos expertos han hecho notar la existencia de una diferencia ideológica notable entre lo que se erige como el movimiento eugenésico principal -constituido esencialmente por Reino Unido, Estados Unidos y Alemania en la primera mitad del siglo XX-, y lo que se presenta como un movimiento eugenésico secundario en que se encuentran diversos Estados americanos como México, Brasil, Argentina o Uruguay; y Estados europeos como Suecia o España.

Según aquéllos, tanto la ideología como la política eugenésica del movimiento principal se reducían al estudio de las características biológicas genético-hereditarias, y a la imposición de una legislación fundamentada sobre la preocupación nacional ante una posible degeneración racial motivada por el mestizaje y las relaciones con deficientes mentales. Por el contrario, la ideología del movimiento secundario y su materialización política se centraban en la 
dupla "puericultura-homicultura" en la que predominaba el cuidado de los individuos a través de mejoras en los sistemas sanitario y educativo, con el objetivo de acrecentar el bienestar de la sociedad.

No obstante, en este texto se mostrará cómo en estos movimientos secundarios no tuvo lugar una reforma de la eugenesia y sus presupuestos per se, sino la coexistencia entre dos perspectivas ideológicas o vertientes eugenésicas distintas que permiten hablar de "hibridación del movimiento eugenésico".

\section{Los inicios del movimiento eugenésico}

El origen del movimiento eugenésico se encuentra directamente relacionado con el interés intrínseco a la especie humana de mejorar sus cualidades físico-intelectuales naturales, con el firme objetivo de desarrollar una vida mejor. Estrictamente hablando, los primeros indicios del interés del ser humano por su auto-mejora pueden rastrearse hasta el escrito sumerio denominado Epopeya de Gilgamesh o Poema de Gilgamesh (Anónimo, 2005) fechado en torno a los siglos XXVII-XXV a.C., la cual se erige como la primera muestra literaria de la preocupación del ser humano por su condición mortal y la pretensión de conseguir la inmortalidad propia de los dioses. Tras esto, el rastro nos lleva hasta el conocido Mito de Prometeo (Esquilo, 1993), en el que se muestra la evidente carencia del ser humano de habilidades intrínsecas para sobrevivir a los peligros del mundo externo y la consecución de la mejora de la especie humana con el regalo de Prometeo.

Poemas y mitos aparte, las primeras señales de propuestas teórico-prácticas de mejora humana aplicables a nuestra realidad material se observan en los siglos V-IV a.C. en el seno del sistema educacional espartano denominado agogé, y del sistema de reproducción pactada propuesto por Platón en su obra La República. La agogé -que fue implantada en Esparta durante el siglo V a.C. de mano del legislador Licurgo- se erigía como el sistema de formación definitivo cuyo propósito era desarrollar los mejores ciudadanos posibles con el adiestramiento físico-militar como pilar fundamental: "el objetivo de la agogé masculina era formar a los jóvenes para ser ciudadanos guerreros" (Cartledge, 2009: 138), lo que le ha valido la denominación de experimento germinal de la eugenesia por parte de los expertos del perfeccionamiento humano (Crowther, 1999). Por su parte, la propuesta platónica recogida en el Libro V de La Repú-

\footnotetext{
${ }^{1}$ La puericultura alude al cuidado materno infantil, mientras que el término homicultura señala al cuidado de la vida de los individuos adultos desde antes incluso de su nacimiento hasta su adultez. Desde los estudiosos de la eugenesia se señala que ambos términos también aludían en última instancia al "cultivo científico de seres humanos más sanos".
} 
blica (Platón, 2006: 300-305), alude a la implantación de un sistema de matrimonios pactados entre hombres y mujeres con las mejores cualidades físicopsicológicas también con el objetivo de criar a los mejores ciudadanos posibles, aunque esta vez forjados sobre el conocimiento y no sobre la guerra:

¿Existe cosa más ventajosa para una ciudad que el que haya en ella mujeres y hombres dotados de toda la excelencia posible? (...) Se desprende la necesidad de que los mejores cohabiten con las mejores tantas veces como sea posible y los peores con las peores al contrario; y si se quiere que el rebaño sea lo más excelente posible, habrá que criar la prole de los primeros, pero no la de los segundos.

Tras otras evidencias eugenésicas relativas a la búsqueda de la mejora humana, como la proclamación de la legitimidad del ser humano para mejorar su naturaleza señalada por pensadores como Picco della Mirandola en el siglo $\mathrm{XV}$ o por Francis Bacon a principios del XVII; y la propuesta educativo-cultural de emancipación racional propia a la Ilustración durante el siglo XVIII; el siglo XIX emergería como el siglo de la eugenesia en lo que a fundamento teórico se refiere. El pensamiento de Charles Darwin, especialmente su propuesta de la selección natural y la "supervivencia del más fuerte" en su obra de 1859 El origen de las especies (Darwin, 1979), y su visión de la humanidad como una fase más de la evolución de nuestra especie expuesta años más tarde en El origen del hombre (Darwin, 1980), supusieron la emergencia de la corriente biológico-social conocida como «Darwinismo Social» que a la postre serviría también como uno de los pilares fundamentales del movimiento eugenésico. Grosso modo, esta corriente extrapola las ideas evolucionistas del pensamiento darwinista al ámbito social y cultural humano, de modo que la idea -ampliamente adoptada por la ideología eugenésica principal en forma de racismo y homofobia- de que los sujetos física, intelectual y moralmente más aptos traerían consigo el amanecer de una nueva era para la humanidad tanto en su vertiente biológica como social, era bastante atractiva para el incipiente movimiento eugenésico que pretendería el perfeccionamiento de la especie humana en todas sus facetas (Soutullo, 1997; Mababu, 2009).

Precisamente el pensador interdisciplinar Sir Francis Galton, primo de Darwin, supuso un paso decisivo para la inserción del pensamiento eugenésico en el ámbito científico al establecer en 1883 el término eugenesia como "la ciencia de la mejora del linaje" (Galton, 1907: 17) con el fin de que sus ideales fueran adoptados como una ciencia por derecho propio, y alentado por la idea darwinista de que los individuos mejor dotados tienen mayor probabilidad de sobrevivir, reproducirse y tener una descendencia ventajosa para su adaptación al entorno. Las tesis de Galton relativas a la herencia de caracteres nega- 
tivos y positivos, y a una posible degradación racial como consecuencia de relaciones entre personas "no aptas", dieron lugar en Reino Unido a una corriente eugenésica multidisciplinar que se centró en la protección del ciudadano británico -especialmente inglés y de buena posición social- frente a la amenaza que suponía para su raza mantener relaciones con personas aquejadas de deficiencias genético-hereditarias. Esta corriente cobró fuerza con la aparición de diversas instituciones como la Sociedad de Eugenesia (Eugenics Society) y diarios como el The Eugenics Review, que estudiaban y difundían la eugenesia como una ciencia pura, tal y como soñaba Galton. Acababa de emerger el primer movimiento eugenésico de la historia, el cual abarcaba ámbitos tan diversos como la Ciencia, la Filosofía, la Comunicación, la Medicina o la Política, y tenía un importante radio de materialización. El siglo XX se presentaría sin duda alguna como el siglo de la eugenesia a nivel práctico, gracias al surgimiento de los dos movimientos eugenésicos más conocidos -estadounidense y alemán-, y a su quehacer político con la implantación de importantes leyes eugenésicas con un fuerte contenido racial.

A razón de la extensión del movimiento eugenésico a numerosos y diversos lugares del mundo, expertos en eugenesia como el historiador estadounidense Daniel Kevles (Kevles, 1985) y más tarde el filósofo noruego Nils RollHansen (Roll-Hansen, 1989) han utilizado los términos Reform Eugenics (eugenesia de la reforma) y Mainline Eugenics (línea principal de eugenesia), para distinguir la eugenesia en su vertiente principal representada por los movimientos eugenésicos británico, estadounidense y alemán durante la primera mitad del siglo XX, de la emergente en otros países de América y Europa cuyos presupuestos eugenésicos esenciales se alejaban de los de aquéllos. Esta presunta reforma de la eugenesia (en tanto que cambio ideológico) alude a la búsqueda incesante de la mejora del ser humano -tanto a nivel individual como colectivo- en el estricto sentido de higiene y bienestar social, en detrimento del racismo y la preocupación por la herencia genética tan propia de la línea principal del movimiento eugenésico en el siglo XX. Algunos ejemplos de esta reforma son los representados por la evolución del movimiento eugenésico en Estados de Norteamérica y Sudamérica como México y Brasil, fuertemente relacionados con la noción de "homicultura" y la dupla saludeducación; y de Estados escandinavos como el sueco en que el "Estado de Bienestar" social primó en la motivación eugenésica en el período de entreguerras. Veremos a continuación esta relación entre el racismo y preocupación biológica propia a los principales movimientos eugenésicos, reservando para más tarde la presunta reforma de los ideales eugenésicos en otros movimientos. 


\section{La línea principal del movimiento eugenésico y la protección de la raza}

El primer movimiento eugenésico, el británico, centró su atención desde sus inicios en la cuestión racial y la posible degradación de la descendencia de sus ciudadanos. A este respecto "la nueva ciencia de Galton proporcionaba, en la mente de los eugenistas, los medios estadísticos por los que se podía prevenir la degeneración y la regeneración podría planificarse" (Porter, 1999: 147), y en consecuencia, el ámbito político británico comenzó a movilizarse. En 1904 se creó una comisión denominada Comisión Real Sobre el Cuidado y Control de los Débiles Mentales (Royal Commission On the Care and Control of the Feeble-Minded), para promover la idea de que la reclusión y esterilización de los deficientes mentales lograría mantener el bienestar biológico de los ciudadanos británicos. De este modo, el movimiento eugenésico británico se caracterizaría indudablemente por la protección de la raza británica -así como su mejora- y su lucha contra los deficientes mentales. Esta idea fue ampliamente expuesta en la «Primera Conferencia Internacional de Eugenesia» (1912), la cual tuvo lugar en Londres y estuvo compuesta por personajes ilustres pertenecientes a todo tipo de ámbitos. Winston Churchill que -como cuenta su principal biógrafo Martin Gilbert- nunca ocultó su interés en la ideología eugenésica y su apoyo al movimiento (Gilbert, 2011), tuvo el rol de vicepresidente en la conferencia. El famoso científico e inventor Alexander Graham Bell y el naturalista Leonard Darwin (hijo de Charles Darwin) actuaron como directores, y otras personalidades de cierta importancia como el antiguo presidente de la Universidad de Harvard Charles Eliot, el profesor de Medicina en la Universidad de Oxford Sir William Osler, el filósofo-matemático Bertrand Russell o el Dr. Ignacio Valentí y Vivó (como único representante español) participaron en él y mostraron la actualidad del mensaje eugenésico (Güvercin y Arda, 2008). Como consecuencia, en 1913 se implantó la ley eugenésica más importante del movimiento británico: la «Ley de Deficiencia Mental» (Mental Deficiency Act). La ley tenía como objetivo principal controlar la debilidad mental a través del registro de enfermos mentales y la prohibición de contraer matrimonio con un individuo aquejado de enfermedades de ese tipo. Al tiempo, otorgaba al Ministro del Interior la potestad de incluir en el programa a individuos cuyas circunstancias lo justificaban (pobreza absoluta, carencia de educación...), pese a no sufrir ningún tipo de enfermedad mental. Fue discutida desde todo tipo de ámbitos, no sólo desde el de la biología o la sociedad como parece desprenderse del ideario eugenésico, sino también desde el histórico y el político, tal y como se señala en las diversas discusiones llevadas a cabo en torno a esta problemática entre el conocido pensa- 
dor irlandés George Bernard Shaw² y el escritor británico Gilbert Chesterton (Chesterton, 2012), quien al contrario que aquél, se mostraba abiertamente en contra del perfeccionamiento humano augurado por los eugenesitas y de la Mental Deficiency Act por su metodología a la hora de clasificar a los sujetos como mentalmente deficientes:

Sólo desarrolla los principios de las Leyes de Dementes para aplicarlos a personas que no tienen ni un rastro de demencia (...) Autoriza el encarcelamiento, supuestamente por demencia, de personas a las que ningún médico se atrevería a declarar dementes. Basta que a un médico o a otra persona se les ocurra considerarlas débiles mentales (Chesterton, 2012: 54-56).

Posteriormente en Reino Unido se debatió ampliamente acerca de la posibilidad de llevar a cabo una política de esterilización para combatir la debilidad mental, con la creación de los denominados comités Wood y Brock en la década de los 20 y 30, y con un fuerte apoyo de instituciones eugenésicas y médicas como la Sociedad de Eugenesia, la Asociación Central de Bienestar Mental (The Central Association for Mental Welfare), la Asociación de Hospitales Mentales (Mental Hospitals Association) o el Consejo Nacional para la Higiene Mental (National Council for Mental Hygiene). Ambos comités desarrollaron informes que, en primer lugar, indicaban el número de deficientes mentales residentes en Reino Unido, y que en segundo, apoyaban la esterilización voluntaria de aquellos sujetos que padecieran algún tipo de deficiencia mental (Blacker, 1962; Porter, 1999). A pesar de las presiones por parte del entorno eugenésico al entonces Ministro de Salud Sir Hilton Young para la implantación de la esterilización, su imposición obligatoria en Alemania y la dureza en su cumplimiento hicieron decrecer el entusiasmo del público en general hacia su uso como solución biológico-social (Wittmann, 2004). Para mediados de los 40, cuando el movimiento eugenésico principal cesó, la eugenesia británica no había avanzado nada desde la implantación de la «Ley de Deficiencia Mental»

Sin embargo, y a pesar de que el movimiento eugenésico británico nunca llegó más allá de esta ley, los movimientos estadounidense y alemán significaron un paso de gigante en sus aspiraciones y también en la dureza de sus políticas. Bajo una evidente influencia británica, la preocupación por la degeneración racial del pueblo americano se extendió rápidamente, tanto así que ya a inicios del siglo XX -concretamente en 1907 y 1910- se implantaban en

\footnotetext{
${ }^{2}$ Shaw nunca tuvo ningún reparo en hacer públicas sus ideas eugenésicas. A este respecto, dedicó parte de sus obras -por ejemplo de su famosa obra Hombre y Superhombrea tratar el tema de la eugenesia y a promulgar la legitimidad del ser humano a llevar a cabo un auto-perfeccionamiento.
} 
los Estados de Indiana y California respectivamente, leyes de esterilización dirigidas a enfermos mentales y a delincuentes, es decir, personas cuyos caracteres hereditarios negativos se traspasarían a su descendencia (amenaza genética). La lucha contra los deficientes mentales a través de la esterilización con la protección racial como objetivo, se acabaría institucionalizando en 1927 en el caso Buck v. Bell (Lombardo, 1985). La fundación de la Oficina de Registro Eugenésico (Eugenics Record Office) en 1910 de mano de Charles Davenport, significó la maximización de la investigación y de la propaganda eugenésica en los Estados Unidos. En ella se hizo un concienzudo registro de características biológicas de familias enteras, y Davenport redefinió la eugenesia como "la ciencia de la mejora de la raza humana por mejor reproducción" (Allen, 1997: 78).

En los años venideros y mientras diversas leyes de esterilización eran aprobadas a lo largo del país norteamericano, el sentimiento racista se iba acrecentando gradualmente, y comenzaban a aparecer los primeros presupuestos relativos a la supremacía racial estadounidense. Como muestra fehaciente de ello, puede observarse el éxito de la obra de índole racial del abogado Madison Grant La caída de la gran raza (The Passing of the Great Race) de 1916, la cual acabaría siendo una referencia principal en la lucha norteamericana contra la inmigración. En dicha obra - con una visible influencia del darwinismo social y las tesis maltusianas sobre la población- se señalaba la necesidad de tomar medidas demográficas relativas a la inmigración para acabar con la degradación racial, y medidas eugenésicas directamente relacionadas con la eliminación de caracteres hereditarios negativos o con la proliferación de los caracteres hereditarios positivos. La obra gozó de gran popularidad tanto en Estados Unidos como en Alemania tras su traducción al alemán en 1926.

Este sentimiento racista se materializó en plena época de entreguerras cuando el movimiento eugenésico comenzó a preocuparse por el alarmante incremento de la inmigración en Norteamérica, y la insuficiencia de las políticas eugenésicas negativas llevadas a cabo hasta ahora para salvaguardar la raza estadounidense: "muchos seguidores estadounidenses de la eugenesia pensaron que las leyes de la restricción de matrimonios y los programas de esterilización serían inútiles, si se consentía que perdurase la amenaza externa al vigor biológico nacional" (Kevles, 1986: 86). Directamente relacionado a este sentimiento de terror nacional al proceso sorprendentemente rápido de reproducción del inmigrante -respecto al ciudadano norteamericano medio-, y sus características hereditarias presuntamente defectuosas, se estableció una de las leyes más importantes en materia eugenésica de la historia de los Estados Unidos: el Acta de Inmigración de abril de 1924 -también conocida como Ley 
Johnson-Reed- que restringía la entrada de inmigrantes procedentes del sur de Europa por su condición médico-social ante la creencia popular y científica de que las características físicas, psicológicas, morales y sociales de los individuos provenientes de Europa del Este eran inferiores a las de los norteamericanos:

En EE.UU. la mayor cantidad de migrantes eran refugiados económicos, pobres, analfabetos y con problemas de salud (...) Pensaban que la falta de oportunidades, los bajos sueldos, la vivienda inadecuada y los problemas sanitarios que conducían a la mayoría de las poblaciones marginadas - entre ellos los migrantes- a la pobreza, la enfermedad y los actos criminales, no eran un problema social, sino de herencia genética y, por tanto, si se podía evitar que estas personas se reprodujeran, entonces el crimen, la pobreza y la enfermedad se eliminarían (Linares Salgado, 2011: 192).

De este momento en adelante, las nuevas leyes de esterilización afectarían también a numerosos inmigrantes al ser considerados erróneamente como deficientes mentales, especialmente por cometer errores de compresión o entendimiento relativos al desconocimiento del idioma o de la cultura americanos. La situación empeoró tras la caída de la banca en 1929 y el contexto social-económico de crisis estadounidense resultante -el cambio de la sociedad rural a la industrial, desempleo, improductividad y recesión-, en que los inmigrantes aparecían como el primer enemigo a batir:

Al público se le dio la clara impresión de que gran parte del conflicto estaba siendo agitado por organizadores inmigrantes que se habían infiltrado en la fuerza de trabajo estadounidense y que estaban trayendo caminos extraños y perjudiciales a una clase laboriosa básicamente pacífica (Allen, 1997: 83).

Esta preocupación también había llegado al otro lado del Atlántico, fundándose en 1925 la Liga Alemana para la Regeneración Nacional y la Herencia, para concienciar de la importancia de la ciencia en la salud de las generaciones futuras con una orientación ciertamente nacionalista; y en 1927 el Instituto Kaiser Wilhelm de Antropología, Herencia Humana y Eugenesia, para dar argumentos científicos que ampararan las políticas raciales que el gobierno alemán pudiera implantar. Cada vez se hacía más patente la aceptación de la idea estadounidense acerca de la necesidad de acabar con los caracteres genéticohereditarios negativos: "después del colapso del estado de bienestar, las medidas eugenésicas negativas fueron vistas como un método con el cual controlar el gasto y salvar a la nación de una crisis genética" (Wittmann, 2004: 19). La entrada del partido nacionalsocialista al gobierno no hizo más que confirmar las ideas eugenésico-raciales del movimiento alemán: 
Vieron [los eugenistas de todas partes del mundo] el ascenso de Hitler como una oportunidad para hacer su nación. "El primero en la historia del mundo" en aplicar "los principios de la raza, genética y selección a la política práctica" (Harvard Facing History Project, 2002: 248).

El movimiento eugenésico alemán se desarrolló en tres pasos: 1) En enero de 1934 con la implantación de la Ley sobre la prevención de la progenie con enfermedades hereditarias, a través de la esterilización de personas tanto alemanas como extranjeras con una genética considerada como no apta; 2) En septiembre de 1935 con el establecimiento de la Ley para la protección de la sangre alemana y el honor alemán, perteneciente a las Leyes de Núremberg y con la que se prohibían los matrimonios entre personas aptas -alemanes puros pertenecientes a la raza aria- y personas no aptas -personas genéticamente deficientes y de razas consideradas inferiores como la judía o la africana-; y 3) Entre los años 1940 y 1942 con la creación del programa de eutanasia obligatoria para personas improductivas Aktion T4 y la aplicación de la Solución Final del Problema Judío que acabó con la vida de más de 6 millones de judíos. La entrada en la Segunda Guerra Mundial y el descubrimiento de los experimentos eugenésicos del gobierno nazi, supusieron el fin del movimiento eugenésico en Alemania y una irremediable desaceleración en movimientos que prosiguieron su quehacer tímidamente como el estadounidense. En éste el racismo biológico seguía vigente, como evidencia el caso Madrigal v. Quilligan durante los años 70 en que diversas inmigrantes mexicanas fueron esterilizadas de forma involuntaria, ante los prejuicios racistas de un médico -James Quilligan-y la legitimación de éstos por parte del Estado de California (Stern, 2006).

Sin embargo, a causa del desarrollo del conocimiento científico-genético, y de los pobres resultados de las políticas eugenésicas para "limpiar" la sociedad de individuos no aptos, -se comenzaba a atisbar que herramientas tales como la esterilización "no cumplían la promesa de solventar con rapidez el problema de la deficiencia mental"- (Kevles, 1986: 142), el movimiento eugenésico principal vio cómo sus ideales sufrían un cambio en sí mismos a pequeña escala ${ }^{3}$ y en el seno de diversos Estados pertenecientes a un movimiento eugenésico secundario a gran escala. De este modo, de forma paralela al movi-

${ }^{3}$ Como bien observa Kevles, los presupuestos eugenésicos británicos y sobre todo estadounidenses sufrieron un cambio en su fundamentación biológico-racial, al sustituir la propaganda racial por la propaganda médico-social, como evidencia el discurso del movimiento norteamericano en las décadas de los 50,60 y 70. No obstante, es probable que este cambio esté más relacionado con la necesidad de separar los ideales eugenésicos estadounidenses -históricamente compartidos con el Tercer Reich- de los alemanes que con un cambio ideológico real, como muestran diversos casos de esterilización con motivación racial durante aquella época. 
miento eugenésico principal, surgieron toda una serie de movimientos eugenésicos en otros países de América y Europa que parecían abandonar las tesis biológico-raciales propias de la línea principal de la eugenesia, y adoptar una ideología basada en la búsqueda del bienestar social propio de los Estados de Bienestar.

\section{La línea reformista del movimiento eugenésico y la búsqueda de bienestar}

Cuando se habla de eugenesia de la reforma, se alude a movimientos eugenésicos -especialmente escandinavos y latinoamericanos- cuyos presupuestos ideológicos se alejan de los habituales biológico-raciales, adoptando unos nuevos relativos a objetivos propios del Estado de Bienestar como la "buena salud" y la "buena educación" 4 . Uno de los principales pensadores que defienden el desarrollo de tal cambio ideológico en el movimiento es el historiador Daniel Kevles, quien en su obra In the Name of Eugenics (1985) hablaba sobre los "eugenistas de la reforma" en los siguientes términos:

Los eugenistas de la reforma se sintieron obligados a romper con la identificación de la habilidad innata con la raza o la clase (...) También argumentaron la importancia, tanto para la eugenesia como para el bienestar social, de una dieta adecuada, del cuidado de la salud, de la vivienda y la educación. Pidieron la abolición de los barrios marginales, la creación de viviendas dignas y de centros recreativos y de guardería, el derecho a un trabajo y un salario justo (Kevles, 1985: 174).

Con el objetivo de analizar la esencia de la eugenesia de la reforma, se analizarán algunos de los movimientos eugenésicos que adoptaron los preceptos médicos y sociales del Estado de Bienestar, concretamente, los movimientos sueco, brasileño y mexicano. En primer lugar y en lo que al movimiento sueco se refiere, ya entre los años 1909 y 1910 se fundaba la Sociedad Sueca de Higiene Racial (Svenska Sällskapet för Rashygien) y la Sociedad Mendel (The Mendelian Society) respectivamente, como herramientas de profusión de la eugenesia con un fuerte contenido racial y que gozaron de bastante éxito antes y después de la Gran Guerra, en tanto que prometían resolver problemas relativos a la inmigración y a los individuos asociales a través de prácticas eugenésico-médicas como la esterilización:

\footnotetext{
${ }^{4}$ Es interesante mencionar que el concepto de salud sufrió un cambio fundamental el 22 de julio de 1946 cuando la OMS declaró en la firma de su constitución que "la salud es un estado de completo bienestar físico, mental y social, y no solamente la ausencia de afecciones o enfermedades". Esta definición señala, por tanto, que en la salud de una persona interviene de forma irremediable el aspecto social de su vida, y en consecuencia el pilar fundamental de la eugenesia reformista era la mejora de las condiciones de vida, tanto a nivel médico (corporal y mental) como a nivel social y cultural (educación, trabajo, vivienda).
} 
La cuestión de la esterilización voluntaria y obligatoria también se planteó por primera vez en este período no sólo por los eugenistas y los científicos genéticos, sino por los responsables políticos y administradores y reformadores sociales interesados en la gestión social de diversos grupos, como los delincuentes y los retrasados mentales (Porter, 1999: 145).

Algunos buenos ejemplos de este interés anterior y posterior a la Primera Guerra Mundial, y que muestran la diferencia entre una eugenesia biológicaracial y una eugenésica del bienestar, son los representados por la Ley sobre el Matrimonio y el Divorcio de 1915 y la creación en 1922 del Instituto Estatal de Biología Racial (Statens Institut för Rasbiologi); y el pensamiento de la diplomática sueca Alva Myrdal -quien fue ganadora del Premio Nobel de la Paz en 1982- y su marido Gunnar Myrdal -quien ganó el Premio Nobel de Economía en 1974-. En primer lugar, la ley sobre el matrimonio fue presentada por el ministro de justicia Berndt Hasselrot en 1911 e implantada en 1915. En virtud de ésta se prohibía el matrimonio a personas con deficiencias mentales y/o con enfermedades infecciosas, al tiempo que estar casados con un individuo que las padeciera serviría como motivo legal para llevar a cabo un divorcio. Posteriormente en 1922 el parlamento sueco aprobó la creación de Instituto Estatal, con el objetivo último de establecer una clara distinción antropológica -a través de un amplio estudio racial de las características de los ciudadanos suecos, de su ascendencia y su descendencia, y especialmente centrado en personas consideradas asociales como los bastardos, los gitanos, los homosexuales o personas con deficiencias físicas y mentales-, entre la raza sueca y otras como la africana o las propias de Europa del Este. Ambos ejemplos se presentan como la máxima expresión del racismo eugenésico en Suecia:

Por la higiene racial de hoy en día, la eugenesia exige cada vez más una legislación que salvaguarde a las generaciones futuras y mejore la raza humana. Este movimiento tiene como objetivo luchar no sólo contra los peligros para la salud pública, creados por fenómenos como la emigración, el industrialismo o la acumulación de personas en las grandes ciudades, sino también los venenos de raza, como la sífilis, la tuberculosis y el alcohol, y por consiguiente, alentar a la sociedad a aumentar el índice de matrimonio en sus grupos mejor calificados; Impidiendo la propagación de los impropios (Thorsten Sellin, 1922: 59).

Por su parte, en 1934 y en el seno de su obra Crisis en la cuestión de la población (Kris i befolkningsfrågan), los Myrdal defendieron fuertemente un tipo de eugenesia positiva directamente relacionado con la maximización del bienestar de pueblo, y no con la ideología racista de los movimientos eugenésicos principales. Propusieron mejoras en los subsidios infantiles y la atención 
sanitaria tanto materna como general, señalando sus efectos deseables sobre la población sueca ante la disminución de la tasa de natalidad en el país: "los Myrdals afirmaron que la disminución de las cifras de cría debía combatirse activamente con medidas positivas de bienestar, o bien la integridad cultural y social del pueblo sueco se vería amenazada" (Spektorowski, 2004: 93). La reforma de los presupuestos eugenésicos en los años 30 alcanzó todo tipo de ámbitos en Suecia, y desde el ámbito político no se tardó en actuar.

La integración del denominado "Estado de Bienestar" -en el estricto sentido de modelo estatal de organización social basado sobre la igualdad de derechos de los ciudadanos y la búsqueda de su bienestar médico-social- vino caracterizada por la inclusión del concepto de Hogar del pueblo (Folkhemmet), esto es, la creación de un Estado sueco por y para la igualdad del pueblo en cualquier faceta de la vida (económica, médica, social, política...), en detrimento del ideal racial de los primeros años de la eugenesia sueca: "el discurso de la eugenesia evolucionó de uno racial y biológico a uno social" (Mizrachi y Spektorowski, 2004: 343). En esta sociedad del bienestar que se vislumbraba desde los ámbitos científico, médico y político, los denominados "asociales" -en sentido estrictamente de improductivos para la sociedad- no tenían cabida: "consideraban a la nación sueca como un todo orgánico, en el que no había lugar para los vividores y los parásitos" (Lucassen, 2010: 273). Como medida legal para acabar con los improductivos, el 1 de enero de 1935 se implantó en Suecia una ley de esterilización con carácter voluntario dirigida a enfermos mentales, marginados sociales y pervertidos, con el objetivo de proteger la población sueca en el más puro sentido de protección social y expansión de la calidad de vida del pueblo sueco. No obstante y a pesar de la voluntariedad otorgada por la ley a los individuos para someterse a la práctica esterilizadora, una gran parte de aquellos que la sufrieron se presentaron voluntarios tras una presunta coacción por parte del Estado motivada por la pretensión de mejorar el bienestar social de la nación (Lennerhed, 1997; Lucassen, 2010). Asimismo la ley establecía un tipo de esterilización obligatoria ciertamente restringida para enfermos mentales con pronóstico grave e irreversible, aunque en 1941 se introdujo un proyecto de ley que expandía el sujeto de esterilización, según el cual pasaría a ser legitimo esterilizar de forma obligatoria a todo individuo considerado "incompetente" o que no llevara una vida acorde con el Estado de Bienestar, fundado en el individuo productivo (el apto) que se pretendía instaurar bajo el yugo de la denominada «Eugenesia del Bienestar» (Mizrachi, y Spektorowski, 2004). Para el año 1976 en que la ley de esterilización de 1935 fue derogada, más de 62.000 personas habían sido esterilizadas, lo cual representa un buen porcentaje del pueblo sueco si se 
tiene en cuenta que su población total ascendía por aquel entonces a 6 millones de personas.

En segundo lugar, el movimiento eugenésico en Brasil se constituye según algunos estudiosos de la eugenesia como una variante esencialmente distinta respecto a lo que venía siendo habitual en línea principal del movimiento eugenésico (Stepan, 1991). En los inicios del movimiento brasileño, predominó la idea subyacente del darwinismo social de que la eugenesia serviría como herramienta para solucionar problemas, tanto de carácter social como el alcoholismo, como de carácter biológico relativos a la raza. En este sentido, la política eugenésica racial y social brasileña se caracterizó por la denominada "Política de Blanqueamiento" (Politica de Branqueamiento), cuyo objetivo fundamental era el decrecimiento de la población negra -relacionada con los esclavos africanos de finales del S. XIX- en favor del crecimiento de la población blanca -asociada a individuos biológica, cultural y socialmente superiores-, ante la preocupación de las taras hereditarias provenientes de los inmigrantes y los esclavos:

Los brasileños se vieron a sí mismos como una gente racialmente mezclada y en gran parte de piel oscura, producto de generaciones de cruces entre indios, africanos y europeos (...) Brasil se consideraba un ejemplo primordial de la "degeneración" que se produjo en una nación tropical racialmente mixta (Stepan, 1991: 44-45).

La extendida preocupación desde los inicios del movimiento eugenésico acerca de la degradación racial, se vio acrecentada en Brasil con motivo de la creación de la Sociedad de Eugenesia de Sao Paulo (Sociedade Eugênica de São Paulo) en 1918, y de la Liga Brasileña de Higiene Mental (Liga Brasileira de Higiene Mental) en 1923. Sin embargo, la década de los 30 trajo consigo otra tendencia eugenésica que adoptaba una ideología más interesada en una mejora de la población brasileña a nivel médico-social, y caracterizada por el cuidado de la salud biológica-social de los individuos desde su niñez hasta su etapa adulta (homicultura); que en la ideología habitual del movimiento eugenésico principal centrada en la lucha contra la degeneración genético-racial: "la educación para la salud de los niños se convirtió en la contraparte esencial de la inspección médica a partir de los años veinte y treinta, con los niños como un conducto para la salud de la familia" (Birn, 2007: 687). La idea fundamental era que la genética humana se ve influida por el ambiente, de modo que implantar medidas higiénicas dirigidas a la salud -mejora de las necesidades básicas, mejora del sistema de salud pública o mejora del sistema educativo- se presentaba como la metodología más oportuna. De lo que se trataba en definitiva era de llevar a cabo una mejora de las condiciones de vida direc- 
tamente relacionada con el lema principal del estado brasileño de Ordem e Progresso (Orden y Progreso):

La gran aceptación del movimiento eugenésico se debió a la promesa de un "nuevo orden social", visto por la clase alta brasileña como el poder de la ciencia que daría "orden y progreso" al país (...) para muchos científicos, políticos y médicos, el movimiento eugenésico era visto como una nueva rama de la higiene y fue lo que permitió a los brasileños reclamar "sanear é eugenizar" [sanear es eugenizar] (Linares Salgado, 2011: 193).

Las medidas estaban pensadas para los individuos de clase social más baja y que carecían de los medios necesarios para tener una salud biológica-social satisfactoria. Desde el sector de la medicina, doctores como Juliano Moreira abogaron por la proclamación del bienestar de la familia y la mejora de sus condiciones de vida: "los pobres del interior rural no eran vistos como amenazas sociales, sino como lamentables, y sus problemas evitables por medio de mejoras en la salud pública y la higiene mental" (Block, 2002: 9). Finalmente el movimiento eugenésico brasileño, como casi todos, se difuminó en los años que siguen al final de la Segunda Guerra Mundial.

Por último y en lo que respecta al movimiento eugenésico en México, éste se inició con el fin de la Revolución Mexicana en 1917 y la preocupación resultante acerca de las características que habría de tener el nuevo pueblo mexicano. En esta visión del futuro pueblo, la eugenesia tenía tres propuestas fundamentales: 1) Higiene racial, fundamentada sobre la idea de blanqueamiento tan extendida en Brasil; 2) Puericultura, vertebrada sobre el cuidado biológico y educacional de los niños; y 3) Prevención sanitaria, basada en el establecimiento de procedimientos o medidas de prevención de enfermedades. En el mismo 1917 se implantó la Ley sobre Relaciones Matrimoniales, la cual establecía que el matrimonio debía regirse según el beneficio de la especie y señalando objetivos manifiestamente eugenésicos como la buena salud en la lucha contra la expansión hereditaria de deficiencias o enfermedades:

El Estado mantiene una preocupación permanente por legislar en torno a las relaciones matrimoniales, con la finalidad de no perpetuar daños físicos, derivados de sífilis, tuberculosis y otras enfermedades que se consideraban contagiosas o hereditarias (Suárez y López Guazo, 1999: 54).

Como añadido, la ley establecía en el artículo cuarto del primer capítulo, la posibilidad de pedir como requisito previo al matrimonio un certificado médico, que demostrara que ninguno de los contrayentes padecía ningún tipo de enfermedad o defecto como los antes mencionados. No obstante, a finales de los 
años 20 comenzó a extenderse un ideal eugenésico basado en el bienestar y la salud, fundándose la Sociedad Mexicana de Puericultura (1929) para promocionar la importancia de la educación sexual, el control de la natalidad y el cuidado del niño. Al tiempo, la Sociedad Eugénica Mexicana (1931) prosiguió este mismo objetivo y gozó de apoyo estatal:

El Estado emprendió una serie de campañas sanitarias y educativas enfocadas, principalmente, a los sectores más vulnerables de la población (...) La eugenesia se sustentaba en argumentos científicos con el fin de perseguir y controlar los llamados "males sociales" de la época, como fueron considerados, por ejemplo, la prostitución o el alcoholismo. La educación en materia de higiene y salud conformó una de esas estrategias, dándose a conocer a través de las escuelas, o bien, por medio de los medios masivos de comunicación. Este tipo de propaganda promovía la higiene doméstica y escolar para que los mexicanos aprendieran a "vivir bien" a través de una "perfecta salud" (Alfaro Gómez, 2012: 108).

A este respecto, en 1932 se implantó una medida eugenésica enfocada a la higiene y bienestar social denominada Proyecto para la educación sexual y la profilaxis de las enfermedades venéreas, a través del cual se pretendía fomentar la educación reproductiva de los niños tanto en el seno de su propia familia, hasta dentro del sistema educativo oficial:

Dado que los inicios de la educación sexual se imparten en el seno de la familia y fundamentalmente por parte de la madre, es indispensable que el estado ofrezca educación sexual para los padres en primera instancia, y dado que ésta debe ser una de las bases de la instrucción moral de la vida escolar, el maestro, el pedagogo, debe brindar educación en torno a los aspectos relacionados con la sexualidad y mantener al mismo tiempo en sus discípulos una sana curiosidad, un verdadero espíritu inquisitivo y amor al conocimiento (Suárez y López Guazo, 1999: 77).

Finalmente y como ocurrió en la mayoría de los movimientos eugenésicos coetáneos, el movimiento eugenésico mexicano perdió fuerza en las décadas que siguen al fin del Tercer Reich, debido a que la eugenesia ya no resultaba atractiva para los objetivos médicos del país, especialmente tras los crímenes que se habían cometido en Alemania bajo su estela.

\section{Hibridación eugenésica}

Si bien es cierto que diversos movimientos eugenésicos se presentan como abiertamente distintos a los de la línea principal de la eugenesia en lo que a sus presupuestos se refieren, en aquéllos existía de forma paralela una corriente de estudio y aplicación eugenésica que seguía ideales biológico-raciales, por lo 
que parece legítimo hablar de "hibridación eugenésica"5 y no tanto de "eugenesia reformista". Pese a que se hable de eugenesia de la reforma en Suecia, y que se entrevea un abandono de los ideales biológico-raciales de los movimientos eugenésicos principales en favor de la lucha por el bienestar de los ciudadanos suecos, en realidad esta lucha estaba fundamentada en unos ideales racistas que se enmascaraban terminológicamente. De este modo, el término "incompetente" fue directamente relacionado con la etnia tattare, siendo señalados junto a los deficientes mentales como los objetivos perfectos de cara a una intervención esterilizadora: "en 1941, durante los debates sobre la revisión de la Ley de 1935, los diputados del Riksdag vieron en la 'nómada' tribu [tattare] 'morena' devastada por la embriaguez, la violencia y el crimen, los candidatos perfectos para la esterilización" (Zylberman, 2004: 919).

En las décadas siguientes aún podrían observarse vestigios de los ideales eugenésicos racistas y homofóbicos. En los años 50 las esterilizaciones fueron reducidas de forma considerable debido a las noticias filtradas por los medios de comunicación que indicaban que diversas personas "habían sido esterilizadas por lo que se consideraban razones equivocadas" (Lind, 2008: 13). En 1972 se introdujo una nueva ley de esterilización manifiestamente homófoba dirigida a las personas que querían cambiar de sexo, siendo la esterilización un requisito previo a la intervención. Además de ello, se abriría una investigación para dilucidar si estos individuos habían donado semen/óvulos, o si los habían congelado para tener descendencia con posterioridad. En tal caso no podrían cambiar de sexo legalmente. Aunque en teoría la legitimidad del Estado para

\footnotetext{
${ }^{5}$ Hay otros movimientos eugenésicos, como el soviético o el español, en los que también tuvo lugar la "hibridación eugenésica" defendida en este texto. En cuanto al primer caso, la ideología eugenésica soviética distinguió en todo momento entre el aspecto racial (diferencias biológico-genéticas entre razas) y el aspecto social (organización de la sociedad, organización económica, organización laboral), aunque ambas vertientes funcionaban y cooperaban con un objetivo común: la creación del nuevo hombre y sociedad soviética. Algunos de sus principales representantes fueron los expertos en genética Yury Filipchenko y Nikolai Koltsov. En lo que respecta al movimiento español, tuvo lugar una íntima relación entre la ideología eugenésica más racial de lucha contra la degeneración de la raza española -visible en el pensamiento del psiquiatra militar José María Vallejo Nágera-, y la ideología eugenésica del bienestar social -defendida por el doctor Gregorio Marañón entre otros- que abogaba por la mejora del cuidado materno y de la educación. Al igual que en el caso soviético, la protección y mejora de la raza (tanto en sentido biológico como social) era el objetivo común de las distintas ramas del movimiento eugenésico. Sin embargo, y en la medida en que la actividad del movimiento soviético como tal se redujo a la década de los 20 (en los 30 fue opacada tras el ascenso de Stalin), y que el movimiento español tampoco se tradujo en medidas políticas concretas o en un discurso social contundente, se ha optado por analizar los movimientos sueco, brasileño y mexicano en los que el desarrollo del pensamiento eugenésico tuvo una mayor transcendencia.
} 
controlar la natalidad mediante la coerción había acabado, la ley de esterilización "transgénero" seguía vigente aún en el año 2012, siendo finalmente derogada en enero del año siguiente. En marzo de 2017 fue propuesta desde el gobierno sueco una ley de indemnización dirigida a los individuos que fueron esterilizados por motivos de cambio de sexo entre los años 1972 y 2013 (más de 800 personas). Otra muestra de que los ideales raciales y homófobos del movimiento eugenésico principal seguían presentes en la presunta eugenesia de la reforma sueca, es que hasta 1997 el quehacer de su política eugenésica fue mantenido en secreto. Precisamente en ese año y en el seno del diario Dagens Nyheter, el periodista Maciej Zaremba publicó los informes sobre las leyes de esterilización impuestas desde los años 30 (Gallagher, 1998), y aunque en un principio ni los gobiernos ni la comunidad médica quisieron hacer declaración alguna al respecto, el gobierno sueco se apresuró a formar una comisión interdisciplinar en que médicos, abogados e historiadores tratarían de dilucidar los "antecedentes políticos y las responsabilidades de los gobiernos y los médicos, estimando el número de esterilizaciones forzadas para hacer propuestas de compensación" (Zylberman, 2004: 917). Por otro lado, y al igual que ocurría con el caso sueco, el ejemplo del movimiento eugenésico brasileño no muestra una reforma de la eugenesia y sus ideales, sino la coexistencia de dos perspectivas eugenésicas contrarias -una estrictamente biológica-racial y otra enfocada al bienestar social-, en la que la segunda acabó predominando en Latinoamérica, entre otras cosas, por su bajo nivel de bienestar respecto a lo que estrictamente se conocía como "Primer Mundo":

Aunque las ideas defendidas por algunos miembros del movimiento eugenéstico se extendieron a medidas más radicales asociadas con la "eugenesia negativa", la mayoría de los eugenistas brasileños se preocupaban por la regeneración de la población del país a través de reformas sociales, ya sea a través del saneamiento urbano y rural, la lucha contra las principales enfermedades del país, la ampliación de la atención materno-infantil o la promoción de la higiene y la educación sexual (Souza et al., 2009: 766).

Ahora bien, que el discurso eugenésico relativo a medidas de bienestar predominara sobre el biológico racial no demuestra que se llevara a cabo una reforma del movimiento eugenésico brasileño, sino que ambos discursos coexistieron teniendo lugar una hibridación de ambas ideologías eugenésicas. En este sentido tuvo lugar en 1929 el Primer Congreso Brasileño de Eugenesia (Primeiro Congresso Brasileiro de Eugenia) en la Facultad de Medicina de Río de Janeiro, en el que se tuvo en cuenta las preferencias de las dos perspectivas eugenésicas existentes en Brasil, a la hora de alentar el interés y promulgar la asunción de la eugenesia como ideología central en los ámbitos científico, 
social, político y académico brasileños, independientemente de si se trataba del pensamiento eugenésico en su vertiente racial o en su vertiente social (Souza et al., 2009). Pese a que había entrado con fuerza la perspectiva eugenésica del bienestar en los años 30, aún a finales de éstos y principios de los 40 , médicos de renombre como el doctor Renato Kehl -denominado como "padre de la eugenesia brasileña"- seguían apoyando firmemente los presupuestos eugenésicos biológico-raciales. A este respecto fundaría el denominado "Boletín de Eugenesia" (Boletim de Eugenia) en 1929 y más tarde escribiría toda una serie de obras -como Lecciones de Eugenesia (1929) y Padres, Médicos y Maestros: los problemas de la educación (1939)- apoyando medidas radicales de eugenesia negativa, especialmente en cuanto a la esterilización se refiere y hasta el final del movimiento eugenésico en Brasil.

Por último y en lo que respecta al caso mexicano, si bien es cierto que predominaron los ideales eugenésicos de salud y bienestar, los ideales raciales estuvieron igualmente vigentes, tanto así que las medidas de salud y educación estaban en muchos casos enfocadas a "mejorar la raza nacional" (Manrique, 2016: 3). Como muestra de ello, en 1931 se fundó la Sociedad Mexicana de Eugenesia para el Mejoramiento de la Raza, formada por profesionales de diversa índole-Ciencia, Medicina, Educación o Política- como representante principal de un movimiento eugenésico mexicano híbrido, en el que coexistían dos vertientes eugenésicas distintas representativas de las dicotomías eugenesia negativa-positiva y protección racial-social.

En una de estas vertientes se defendía un ideal biológico de "raza mestiza" superior a la raza mexicana convencional, fundamentada en la creencia de que el mestizaje otorgaba al individuo mexicano un equilibrio perfecto entre resistencia física y desarrollo psicológico-espiritual (Gómez Fröde, 2013). Uno de sus mayores defensores fue el conocido filósofo mexicano José Vasconcelos, quien en su obra La raza cósmica (1925) afirmó que la mezcla de razas "conducirá a la formación de un tipo infinitamente superior a todos los que han existido" (Vasconcelos, 2007: 29), es decir, que el mestizaje -especialmente entre españoles e indígenas- permitirá la aparición de una nueva raza superior. Por su parte, la otra vertiente estaba directamente relacionada con la intervención en todo aquello relacionado con la vida del ser humano más allá de su propia biología, esto es, sus condiciones de vida y desarrollo: higiene, salud, alimentación, educación, familia... en definitiva, los pilares fundamentales de la eugenesia del bienestar. Además, como muestra de la hibridación ideológica del movimiento mexicano, la Sociedad Mexicana creó en el mismo 1931 su propia revista de divulgación eugenésica, a través de la cual difundió todo tipo de información eugenésica afín a cualquiera de las dos vertientes, 
desde cursos sobre salud reproductiva y educación sexual o propaganda relativa a la lucha contra el alcoholismo, hasta medidas más contundentes como la esterilización en la lucha contra los genes defectuosos. Finalmente, y al tiempo que se ponían en marcha proyectos propios de la eugenesia del bienestar tales como la puericultura, se aprobaba en 1932 -concretamente en el Estado de Veracruz- la única ley de esterilización eugenésica de todo México, dirigida a los deficientes mentales, degenerados y delincuentes en virtud de la protección de la raza frente a su degradación.

\section{Conclusión}

El movimiento eugenésico principal -compuesto por Reino Unido, Estados Unidos y Alemania- se fundamentó hasta la caída del Tercer Reich en la discriminación racial y la lucha contra personas con discapacidades intelectuales, en una insaciable búsqueda por la protección de la raza ante la seria amenaza de su degeneración como consecuencia del mestizaje y las relaciones con los genéticamente menos aptos. El movimiento estadounidense, que prosiguió su camino de forma tímida tras los horrores del Holocausto, llevó a cabo un presunto cambio ideológico que no se tradujo en la realidad, como muestra la continuación de su política de esterilización hasta la década de los 70 basada en el rechazo del inmigrante y de sus caracteres hereditarios "indeseables".

De forma paralela al movimiento eugenésico principal emergieron numerosos movimientos en Estados norteamericanos, europeos y sudamericanos -entre los que destacan México, Suecia y Brasil- que constituyeron una línea de eugenesia secundaria. A pesar de que en sus inicios estos movimientos imitaron los ideales eugenésicos procedentes de la línea principal -especialmente los relativos a la degradación racial- surgió en ellos una corriente alternativa que promovía la protección de los ciudadanos a nivel médico-social y cultural con independencia de una motivación de carácter genético-racial.

Sin embargo, no es posible hablar de "eugenesia de la reforma" o de "sustitución de la ideología eugenésica" -como hacen Kevles y Roll-Hansen- si se tiene en cuenta que en estos movimientos eugenésicos pertenecientes a la línea secundaria, la corriente ideológica genético-racista no sufrió cambio o reforma alguno, sino que prosiguió su curso de forma paralela a la nueva corriente eugenésica del bienestar médico-social. Es por ello que pese a la diferencia ideológica y metodológica de ambas perspectivas; la esterilización propia de la lucha contra las deficiencias genéticas achacadas tanto a enfermos físico-intelectuales como a individuos de otras razas, y el cuidado maternoinfantil junto a la mejora de la alimentación y la educación propia de la búsqueda del bienestar médico-social; las sociedades eugenésicas -emergentes en 
la línea de eugenesia secundaria- trataron de promocionar y establecer la eugenesia como herramienta solucionadora de problemas tanto biológicos como sociales, desarrollando un discurso que no marginara ninguna de aquéllas y fortaleciendo la idea de una "hibridación eugenésica" en los movimientos eugenésicos secundarios frente a la idea habitual de reforma.

Finalmente, es interesante observar que si bien la línea más racial del movimiento eugenésico híbrido parece estar lejos de revivir sus días de gloria, todavía hoy puede rastrearse el alcance de la eugenesia del bienestar -en ocasiones bajo el nombre de "nueva eugenesia" o "eugenesia liberal"- en el seno de los avances tecnológicos y la emergencia de técnicas biomédicas desde la década de los 70 del siglo XX, y especialmente en cuanto a los diagnósticos genéticos -que permiten conocer la información genética de los futuros nacidos desde su etapa de embrión- y la selección embrionaria -elección de un embrión para su implantación en función de dicha información genética- se refiere. Precisamente la posibilidad de escoger no implantar un embrión o de realizar un "aborto terapéutico" -a veces denominado eugenésico- para evitar que el futuro nacido padezca una patología determinada (en busca de su bienestar), ha hecho emerger un amplio debate socio-político y también médico en torno a la utilización de estas herramientas tecnológicas y los posibles problemas ético-sociales derivados de su uso, como puede ser la marginación y discriminación de sujetos que padecen una enfermedad o discapacidad (tan habitual en la vertiente homófoba del movimiento eugenésico). En este sentido, podría ser interesante analizar el impacto de la eugenesia del bienestar en el siglo XXI y sus problemas sociales, tal y como reclaman asociaciones y fundaciones de pacientes con Síndrome de Down o Espina Bífida.

\section{Bibliografía}

ALLEN, Garland (1997). The social and economic origins of genetic determinism: a case history of the American Eugenics Movement, 1900-1940 and its lessons for today. Genetica, 99, 77-88. http://dx.doi.org/10.1023/A:1018396529332

ANÓNIMO (2005). Poema de Gilgamesh. Estudio preliminar, traducción y notas de Federico Lara Peinado. Madrid: Tecnos.

BIRN, Anne-Emanuelle (2007). Child health in Latin America: historiographic perspectives and challenges. História, Ciências, Saúde - Manguinhos, 14 (3), 677-708. http://dx.doi.org/10.1590/S0104-59702007000300002

BLACKER, Carlos (1962). Voluntary Sterilization: The Last Sixty Years". The Eugenics Review, 54 (1), 9-23.

BLOCK, Pamela (2002). Sexuality, Parenthood, and Cognitive Disability in Brazil. Sexuality and Disability, 20 (1), 7-28. http://dx.doi.org/10.1023/A:1015230303621

CARTLEDGE, Paul (2009). Los espartanos. Una historia épica. Barcelona: Ariel. 
CHESTERTON, Gilbert (2012), La eugenesia y otras desgracias. Sevilla: Espuela de Plata. CROWTHER, Nigel (1999). Sports, nationalism and peace in Ancient Greece. Peace Review, 11 (4), 585-589. http://dx.doi.org/10.1080/10402659908426311

DARWIN, Charles (1979). El origen de las especies. Madrid: EDAF.

DARWIN, Charles (1980). El origen del hombre y la selección en relación al sexo. Madrid: EDAF:

ESQUILO (1993). Prometeo encadenado. Madrid: Gredos.

GALLAGHER, Paul (1998). The Man Who Told the Secret. Columbia Journalism Review, $36(5), 65-66$.

GALTON, Francis (1907). Investigaciones sobre las facultades humanas y su desarrollo. Londres: J. M. Dent \& Co.

GILBERT, Martin (2011). Churchill and Eugenics. Finest Hour. The Journal of Winston Churchill, 152, 44-48.

GÓMEZ FRÖDE, Carina (2013). Eugenesia: moralidad o pragmatismo. Gaceta Médica de México, 149 (4), 476-480.

GÜVERCIN, Cemal Hüseyin; ARDA, Berna (2008). Eugenic concept: from Plato to present. Human reproduction ang genetic ethics, 14 (2), 20-26.

HARVARD FACING HISTORY PROJECT (2002). Race and Membership in American History: The Eugenic Movement. Brookline: Facing History and Ourselves National Foundation.

KEVLES, Daniel (1985). In the Name of Eugenics: Genetics and the Uses of Human Heredity. Cambridge: Harvard University Press.

KEVLES, Daniel (1986). La Eugenesia. ¿Ciencia o utopía? Una polémica que dura cien años. Barcelona: Planeta.

KEVLES, Daniel (1992). Controlling the Genetic Arsenal. The Wilson Quarterly, 16 (2), 68-76.

KLAUTKE, Egbert (2016). The Germans are beating us at our own game: American eugenics and the German sterilization law of 1933. History of the Human Sciences, 29 (3), 25-43. http://dx.doi.org/10.1177/0952695116631230

LENNERHED, Lena (1997). Sterilisation on Eugenic Grounds in Europe in the 1930s: News in 1997 but Why? Reproductive Health Matters, 5 (10), 156-161. http://dx. doi.org/10.1016/S0968-8080(97)90098-7

LINARES SALGADO, Jorge; VILLELA CORTÉS, Fabiola (2011). Eugenesia. Un análisis histórico y una propuesta. Acta Bioethica, 17 (2), 189-197. http://dx.doi.org/ 10.4067/S1726-569X2011000200005

LIND, Judith (2008). The best interest of the child as an argument in assessments of parent potential in Sweden. International Journal of Law, Policy and the Family, 22, 1-21. http://dx.doi.org/10.1093/lawfam/ebm014

LOMBARDO, Paul (1985). Three generations, no imbeciles: new light on Buck v. Bell. New York University Law Review, 60 (30), 30-62.

LUCASSEN, Leo (2010). A Brave New World: The Left, Social Engineering, and Eugenics in Twentieth-Century Europe. IRSH, 55 (2), 265-296. http://dx.doi.org/10. $1017 /$ S0020859010000209 
MABABU, Richard (2009). La influencia de Charles Darwin en el estudio de las diferencias individuales, de Francis Galton. Revista de Historia de La Psicología, 30 (23), 215-222.

MANRIQUE, Linnete (2016). Dreaming of a cosmic race: Jose Vasconcelos and the politics of race in Mexico, 1920s-1930s. Cogent Arts \& Humanities, 3, 1-13. http://dx.doi.org/10.1080/23311983.2016.1218316

MIZRACHI, Elisabet; SPEKTOROWSKI, Alberto (2004). Eugenics and the Welfare State in Sweden: The Politics of Social Margins and the Idea of a Productive Society. Journal of Contemporary History, 29 (3), 333-352. http://dx.doi.org/10.1177/00220 09404044443

PLATÓN (2006). La República. Madrid: Alianza.

PORTER, Dorothy (1999). Eugenics and the Sterilization Debate in Sweden and Britain before World War II. Scandinavian Journal of History, 24 (2), 145-162. http://dx.doi.org/10.1080/03468759950115773

ROLL-HANSEN, Nils (1989). Geneticists and the Eugenics Movement in Scandinavia. The British Journal for the History of Science, 22 (3), 335-346. http://dx.doi.org/ $10.1017 /$ S0007087400026194

SOUTULLO, Daniel (1997). La eugenesia desde Galton hasta hoy. Madrid: Talasa.

SOUZA, Vanderlei et al (2009). The National Museum's Physical Anthropology Archives: sources on the history of eugenics in Brazil. História Ciências Saúde-Manguinhos, 16 (3), 763-777. http://dx.doi.org/10.1590/S0104-59702009000300012

SPEKTOROWSKI, Alberto (2004). The Eugenic Temptation in Socialism: Sweden, Germany, and the Soviet Union. Comparative Studies in Society and History, 46 (1), 84-106. http://dx.doi.org/10.1017/S0010417504000052

STEPAN, Nancy (1991). The Hour of Eugenics. Race, Gender and Nation in Latin America. New York: Cornell University Press.

STERN, Alexandra (2006). Esterilizadas en nombre de la salud pública: raza, inmigración y control reproductivo en California en el Siglo XX. Salud Colectiva, 2 (2), 173-189. http://dx.doi.org/10.1590/S1851-82652006000200006

SUÁREZ y LÓPEZ GUAZO, Lara (1999). La influencia de la sociedad eugénica mexicana en la educación y en la medicina social. Asclepio, 51 (2), 51-84. http://dx.doi.org/ 10.3989/asclepio.1999.v51.i2.310

THORSTEN SELLIN, Johan (1922). Marriage and Divorce Legislation in Sweden, Minneapolis.

VASCONCELOS, José (2007). La raza cósmica. México D.F:: Editorial Porrúa.

WITTMANN, Emily (2004). To what extent were ideas and beliefs about eugenics held in Nazi Germany shared in Britain and the United States prior to the Second World War? Vesalius. Acta Internationales Historiae Medicinae, 10 (1), 16-19.

ZYLBERMAN, Patrick (2004). Eugénique à la scandinave: le débat des historiens. Medecine/Sciences, 20 (10), 916-925. http://dx.doi.org/10.1051/medsci/20042010 916 